\title{
AVIATOR - Assessing aViation emission Impact on local Air quality at airports: TOwards Regulation
}

\author{
Victor Archilla ${ }^{1, *}$, Dévora Hormigo ${ }^{1}$, María Sánchez-García ${ }^{1}$, and David Raper $^{2}$ \\ ${ }^{1}$ Turbojet Test Centre, INTA, Ctra. de Ajalvir, km 4, Torrejón de Ardoz, 28850, Spain \\ ${ }^{2}$ Centre of Air Transport and the Environment, Faculty of Science and Engineering, Manchester \\ Metropolitan University, Manchester M15GD, UK
}

\begin{abstract}
Emissions from aircraft have adverse effects on the air quality in and around airports, contributing to public health concerns within neighbouring communities. AVIATOR will adopt a multi-level measurement, modelling and assessment approach to develop an improved description and quantification of the relevant aircraft engine emissions, and their impact on air quality under different climatic conditions. Particulate and gaseous emissions in a test cell and on-wing from an in-service aircraft will be measured to determine pollutant plume evolution from the engine and APU exhaust. This will provide an enhanced understanding of primary emitted pollutants, specifically the nvPM and vPM (down to $10 \mathrm{~nm}$ ), and the scalability between the regulatory test cell and real environments. AVIATOR will develop and deploy a proof-of-concept low cost sensor network for monitoring UFP, PM and gaseous species across multiple airports and surrounding communities. Campaigns will be complemented by high-fidelity modelling of aircraft exhaust dynamics, microphysical and chemical processes within the plume. CFD, box, and airport air quality models will be applied, providing validated parameterisations of the relevant processes, applicable to standard dispersion modelling on the local scale. Working with the regulatory community, AVIATOR will develop improved guidance on measuring and modelling the impact of aircraft emissions, and will provide airports and regulators with tools and guidance to improve the assessment of air quality in and around airports.
\end{abstract}

\section{Objectives}

\subsection{Rationale}

Scientists and regulators have an increasingly informed understanding of the complex nature of Particulate Matter (PM) in ambient air, in terms of particle size and chemical composition from different sources both natural and anthropogenic. What is less certain, is how PM

\footnotetext{
* Corresponding author: archillapv@inta.es
} 
species and precursors evolve and interact within the atmosphere, and which characteristics of the PM are the most harmful to public health [1]. Within this context, it is considered Ultra Fine Particles (UFP) - particles of aerodynamic diameter less than $100 \mathrm{~nm}$ - may have greater toxicity on an equal mass basis than currently regulated larger particles $\left(\mathrm{PM}_{2.5} / \mathrm{PM}_{10}\right.$ ambient standards) because their vast numbers and small diameters provide a high surface area which is a potentially important toxicological interface. These UFP's are relevant to civil aviation, and recent studies have shown aircraft engines emit primary aerosol, such as non-volatile Particulate Matter (nvPM), and secondary aerosol precursor gases, such as organic gases and sulphates [2], that nucleate within the exhaust plume within this size range. The contribution of UFP from aircraft operations to the emission inventory in and around airports is therefore largely unknown, and could be significant [3].

Concern regarding air quality in and around airports has been recognised by The Advisory Council for Aviation Research and Innovation in Europe (ACARE) who identify within Action Area 3.5 [4] the need for airport development to be sustainable, stating "assessment of air quality impact at or near airports must be based on accurate measures or prediction of air vehicle emissions combined with sound atmospheric transport models". These challenges, further elaborated within Flightpath 2050, amplify the societal and industry requirement for an enhanced qualitative and quantitative understanding of aircraft engine emissions, physicochemical interactions and dispersion, with specific reference to pollutants that may have significant health impacts.

\subsection{Aims and Objectives}

AVIATOR will adopt a multi-level measurement, modelling and assessment approach to develop an improved description and quantification of relevant aircraft engine emissions, and their impact on air quality in and around airports under different climatic conditions.

The project Aims and their concrete and measurable objectives are set out as follows:

Aim 1. Aircraft Engine Emission Measurements: To measure, quantify and characterise airborne pollutant emissions from aircraft engines under parking (with functioning APU), taxiing, approach, take-off and climb-out, with specific reference to total ultra-fine particles, $\mathrm{NO}_{\mathrm{x}}, \mathrm{SO}_{\mathrm{x}}$ and VOC under different climatic conditions.

- To develop approaches for measurement protocols at engine exit and downstream within the evolving plume, across a range of operational conditions representative of the Landing Take-Off (LTO) cycle in a well characterised test-cell. Measurements will provide characteristics of the near-field physical and chemical composition of engine emissions; meanwhile, when coupled with the captive nature of the exhaust stack, will allow for ambient conditions on plume evolution to be investigated.

- To measure on-wing main engine emissions over a range of operational and ambient conditions (seasonal and diurnal) to develop an improved understanding of exhaust plume composition and evolution in and around an airport.

- To measure emissions from aircraft engines using certified alternative fuel to investigate its impact on total PM formation and evolution in the plume as well as the wider airport environment.

- To perform measurements of air quality in and around 3 international airports: MadridBarajas, Zurich and Copenhagen, to validate model developments under different conditions, and to develop a proof-of-concept low cost and low intervention sensor network to provide routine data on temporal and spatial variability of key pollutants including UFP, total PM, $\mathrm{NO}_{\mathrm{x}}$ and $\mathrm{SO}_{\mathrm{x}}$.

Aim 2. Aircraft Plume and Airport Modelling: To develop understanding of main engine and APU exhaust plume evolution, dispersion, microphysics and chemistry. To improve the 
ability of existing dispersion models to describe the impact of aircraft emissions on air quality in and around airports.

- To investigate the microphysics and chemistry of pollutant formation and evolution (with emphasising on total PM) from the exit of the main engine and APU to identify limitations of the currently adopted regulatory approach towards an improved representation and parameterisations suitable for use in future dispersion modelling and standard setting.

- To describe the physical dynamics of the main engine and APU exhaust plume to move towards the development of validated parameterisations suitable for future dispersion modelling and standard setting. This will specifically consider the interaction of wing tip vortices during take-off and approach.

Aim 3. Bridging the gap between Aircraft Engine Certification and Local Air Quality (LAQ) Regulation: To establish interdependencies in the air quality causality chain that better link the current engine emission certification methods with local air quality regulation which is designed for health protection.

- To describe the causality between the regulated gaseous and nvPM engine emissions species and the subsequently evolved total PM plume concentrations, to better understand the uncertainties and constraints of current regulation stringencies for LAQ prediction.

- To build on the knowledge gaps and requirements of stakeholders; to develop and agree new outline agendas to benchmark and improve regulation of aircraft emissions that is better aligned to the needs and current understanding of airport air quality.

- To develop understanding of vPM and secondary PM precursor emissions at fleet level and within the context of regulatory development.

Aim 4. Protocols and Guidance for Air Quality and Health Impact Assessment: To develop protocols and sampling techniques for the extraction of representative material suitable for in-vitro and in-vivo analysis.

- To develop protocols and methodologies for the sampling of representative UFP, VOC and SVOC during the test-cell and on-wing measurements of engine emissions for utilisation by relevant health professionals for in-vitro and in-vivo assessment of health effects.

- To provide detailed aircraft exhaust modelling guidance, including validated datasets and parameterisations for modelling and regulatory communities.

- To provide detailed guidance on measuring pollutants at airports with specific reference to UFP and the utility of low-cost sensor networks.

- To provide contribution to ICAO Doc 9889 (Airport Air Quality Manual) via CAEP working group participation, where AVIATOR partners are established members.

- To disseminate and communicate AVIATOR outcomes to the widest possible audience through existing regulatory, industry and community stakeholders.

\section{Methodology}

The novel approach of AVIATOR in delivering the ambitious and challenging science and innovation aims and objectives mentioned is based on:

1.Developing and employing a standardised and traceable high-fidelity measurement approach across different scales: (i) certified test cell engine exit and stack measurements; (ii) on-wing engine (and APU) emissions, and (iii) airport air quality. This will provide important insights into the utility and robustness of in-stack measurements of total PM, being appropriate in describing a new EI for possible use by regulatory bodies and for impact assessments.

2.Developing, validating and demonstrating the value and utility of a low-cost sensor network for airport application to provide temporally and spatially resolved information on the key pollutants, including $\mathrm{NO}_{2}$ and UFP number. 
3.Developing a robust understanding of relevant UFP transformation and processes within aircraft exhaust plumes by employing a novel nested model approach. New parameterisations will be developed to facilitate an improved prediction of UFP number concentrations, and other pollutants in and around airports. A quantitative proof-of-concept for regulatory frameworks will be determined through the application of the new parameterisations within state-of-the-art dispersion models for two major airports validated with high-fidelity measurements.

The intention of AVIATOR is not only to advance understanding of the impact of aircraft emissions on air quality in and around airports with a focus on UFP and precursors (SVOC and VOC), but also to be the benchmark on which regulations to improve airport air quality can be assessed.

\subsection{Test-cell engine exit and in stack plume measurements (WP2)}

Several experiments and measurements will be conducted on large modern Rolls-Royce Trent family development engines in the INTA test cell facility. Analysis of exhaust gases (including aerosol precursors) and aerosol phase (nvPM and vPM down to diameters of at least $10 \mathrm{~nm}$ ) measurements will be conducted at engine exit and 50m downstream in the exhaust stack (positions X1 and X2 in Fig. 1). Engine conditions will simulate the LTO cycle (idle to MTO). Using a test cell to investigate the plume chemistry has a number of advantages over on-wing experiments.

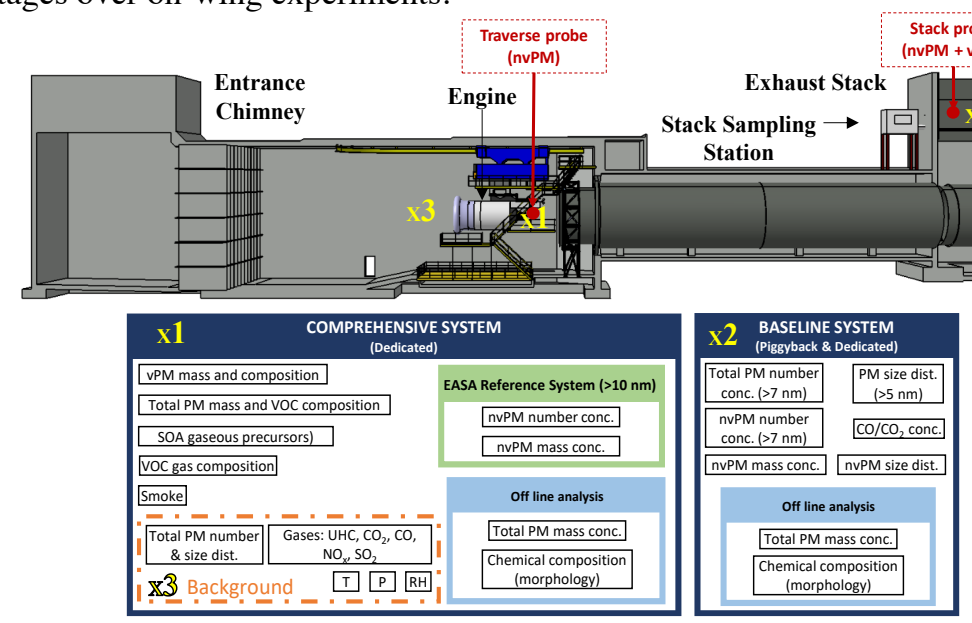

Fig. 1. Test-cell engine emission measurement schematic.

Test-cell data will: (i) ensure uncertainty associated with ambient conditions (temperature, pressure, humidity); (ii) limit the potential effect of solar radiation on plume evolution, and (iii) allow for the impact of local wind conditions on plume advection to be removed. Background measurements will additionally be made at the test-cell inlet to assess the impact of ambient conditions.

\subsection{Test-cell engine exit and in stack plume measurements (WP2)}

This WP will be led by CU in collaboration with the international partner NRC. Emissions measurements will be undertaken to establish concentrations of gases, nvPM and total PM behind both functioning APU and main engines 'on-wing' (Fig. 2), using sampling and highfidelity measurement protocols developed in the test-cell environment. Measurement analysers will be suitably calibrated and inter-compared prior and post-test campaigns. 
The on-wing and plume measurements will take place at IBERIA's service facility (Madrid-Barajas Airport), located 5km from the INTA test cell where WP2 'Piggyback' and Dedicated tests will take place. To establish a better understanding of the evolution of pollutants in the exhaust plume of an aircraft during the LTO cycle, including functioning APU, and the impact of climatic conditions (and potentially solar radiance), two distinct test programmes will be conducted during differing seasons (Season 1, summer; Season 2, winter). Testing will take place at: (i) varying times (day and night); (ii) different aircraft axial locations, and (iii) different APU settings and engine power levels relevant to airport operations (LTO cycle). The influence of fuel composition on emissions will additionally be studied during an 'alternative' drop in fuel test.

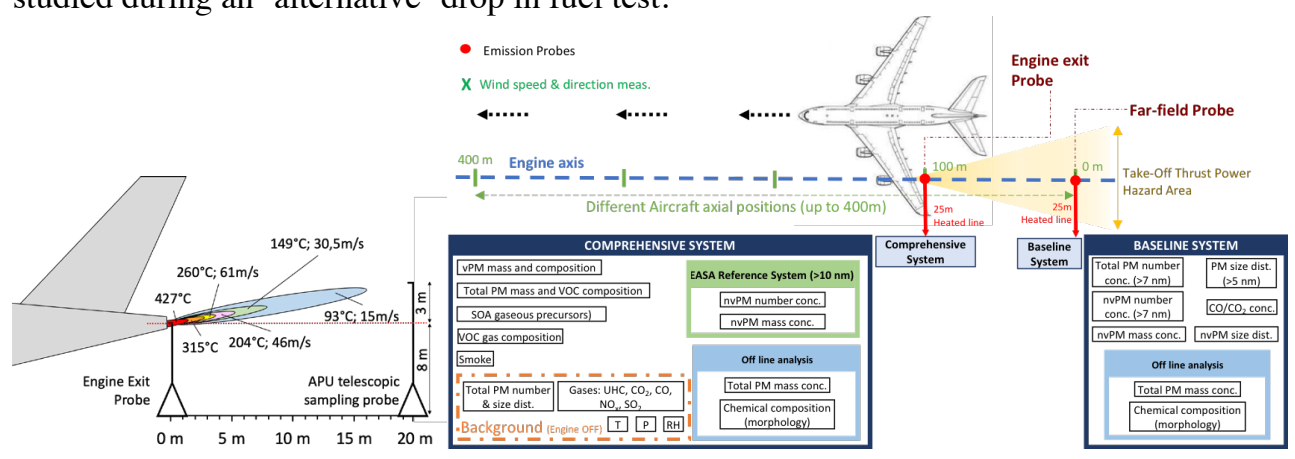

Fig. 2. APU (left) and on-wing (right) test schematics.

\subsection{Ambient measurements and sensor network development (WP4)}

There have been many detailed studies of airport air quality in Europe and elsewhere [5]. To a large extent these have been based on the deployment of research grade, high-fidelity instrumentation that have provided detailed temporal information on pollutants identified in EU air quality Directives [6] and more recently UFP and nvPM. Few studies have provided a detailed investigation of speciated VOC and SVOC and PM specification profiles which are likely to be important for understanding the possible health impact of aircraft emissions. In addition, due to cost, many airport air quality studies have been reliant on a small number of sites which provide little information of the spatial distribution of air pollutants. Therefore, AVIATOR will address these critical knowledge gaps through:

Proof-of-concept low-cost sensor system: AVIATOR will develop a low-cost integrated sensor unit for monitoring key pollutants including $\mathrm{CO}_{2}, \mathrm{CO}, \mathrm{NO}_{\mathrm{x}}, \mathrm{HC}, \mathrm{SO}_{2}$ and UFP, as well as key meteorology. AVIATOR will deploy these nodes alongside high-fidelity measurement equipment at the campaign airports to (i) validate the feasibility of a low-cost measurement approach; (ii) to assess the robustness of the advanced dispersion modelling developed which account for the chemical and physical processes within aircraft plumes; and (iii) to provide a longer-term dataset for the use by scientific and airport regulatory communities.

High-fidelity instrumentation: Deploying a high-fidelity instrumentation system at Madrid Airport to: (i) better characterise air quality in and around airports with specific reference to PM, VOC and SVOC; (ii) validate a proof-of-concept low-cost sensor network; (iii) provide data for model validation; (iv) augment the on-wing measurement campaigns at Madrid. An element of the high-fidelity instrumentation will be additionally deployed at Zurich and Copenhagen to provide additional information on how climate may influence the chemistry of aircraft exhaust and to continue to benchmark the performance of the low-cost sensors. 


\subsection{Modelling of plume microphysics, chemistry and dynamics (WP5)}

Comprehensive aerosol dynamics modelling in AVIATOR will be focus on two aspects relevant for possible health impacts: evolution of size distribution and mixing state of nvPM, and formation and evolution of vPM. In addition, in a set of sensitivity studies, AVIATOR will investigate the ability of UFP to get transported over large distances from the airport. Interaction of aviation-induced aerosols with those from background at varying conditions (e.g. pollution level, relative humidity and other meteorological quantities) will be considered. Engine exit parameters for the jet modelling are gained from WP2 and WP3 measurements data, supplemented as required by inter-engine box modelling [7]. The modelling will be performed using a cascade of different model types, each with their own strengths and limitations (Fig. 3):

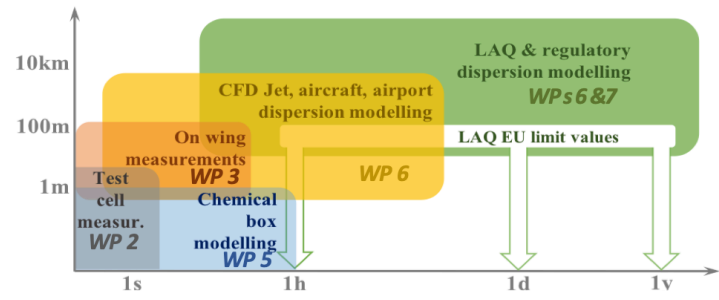

Fig. 3. Temporal and spatial scales covered by AVIATOR models and measurement devices.

A numerical aerosol dynamics and chemical box model [8] (MADE3) will be applied to study the temporal evolution of PM in the exhaust emitted by an aircraft main engine and an APU. CFD jet modelling [9] (CEDRE) will be used to cover the initial hot jet phase after engine exit (up to some $10 \mathrm{~m}$ behind the engine) and to provide input for the chemical box model on plume dilution and plume temperature during the jet phase (up to some $100 \mathrm{~m}$ behind the engine). The CFD modelling considers core and bypass flow, engine geometry based on representative jet engine and an APU, main chemical conversions of gaseous $\mathrm{SO}_{\mathrm{x}}$ as precursor of volatile PM and monodisperse non-volatile PM emission.

For distances beyond the jet phase, plume dilution as an input for the box model will be provided by standard dispersion modelling [10] (LASAT) and classical plume spread estimates. Suitable information on changes in ambient background concentration will be derived from measured data. This will allow to investigate with box modelling the fate of UFP up to a time scale of about one hour with according transport distances of the order of $10 \mathrm{~km}$.

Measurements carried out in WP2 and WP3 will be used to define realistic values for key parameters and to repeat comprehensive model simulations, allowing the verification of key processes as well as the estimation of their importance for impacts on air quality.

As part of the physical dynamics studies, wing vortices formation, their evolution, interaction with the ground, and the trapping of the engine exhaust into the vortices will be studied using CFD aircraft modelling [11] (simulations based on the CAD of an aircraft with CEDRE). Of particular interest are the LTO segments initial climb and approach, where wing vortices may shift part of the pollutant plume towards the ground.

\subsection{Pollutant modelling and transport in and around airports (WP6)}

The approximations and parameterisations developed in WP5 will be implemented into LASPORT and CEDRE dispersion model systems (airport model: aircraft emissions modelled by mobile sources; Fig. 4) and applied as a proof-of-concept in the airport dispersion calculations carried out for the pollutant modelling and transport in and around the airports. 


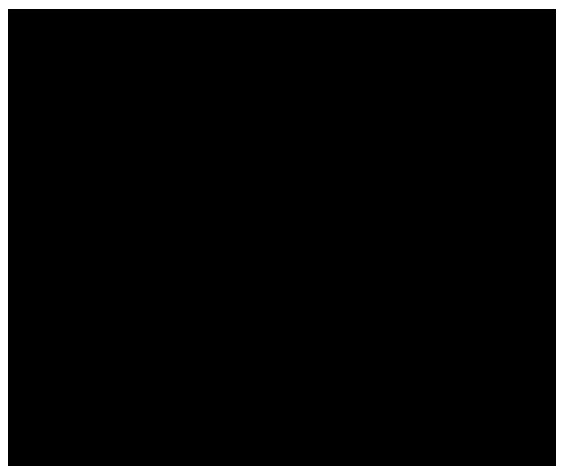

Fig. 4. Calculated nvPM number concentration obtained from LASPORT dispersion model system.

Dispersion modelling provides detailed information on the spatial distribution of pollutant concentration in and around airports and on the contribution of different emission groups. Aircraft main engines and APU are the dominant emission sources for key pollutants like $\mathrm{NO}_{\mathrm{x}}$ and $\mathrm{SO}_{x}$. Recent studies indicate they are the dominant source of UFP, both with respect to mass and number [12], as well. While dispersion calculations for $\mathrm{NO}_{\mathrm{x}}$ and $\mathrm{SO}_{\mathrm{x}}$ are routinely applied, major uncertainties exist for UFP in view of specifying the mass and number emissions and the evolution of volatile UFP during atmospheric transport. Using the findings of WP5, dispersion calculations for $\mathrm{NO}_{\mathrm{x}}, \mathrm{SO}_{\mathrm{x}}$ and UFP emitted by aircraft will be carried out for two airports and compared to concomitant measurements from WP4. This will validate and test the fit-for-purpose of the methodologies developed in WP5 to model the aircraft-related concentration in and around airports.

The modelling will be carried out with the airport dispersion modelling system LASPORT [13] and the CFD model CEDRE. Both models track the individual aircraft movements with a time resolution down to some seconds and account for engine emissions, conversion processes and exhaust dynamics in a time-resolved, parameterised form. The dispersion calculations will provide the 3-dimensional concentration distributions as successive temporal averages (LASPORT 1 hour, CEDRE 5 minutes) for a given period of time (LASPORT several months, CEDRE several hours), spatial resolution (LASPORT down to about 50m, CEDRE down to about $1 \mathrm{~m}$ ) and spatial extent (up to distances of about $20 \mathrm{~km}$ from the airport). Time period and computation grid will be matched with the pollutant measurements carried out at the airports in WP4.

\subsection{Bridging the gap: Aircraft Engine Certification and LAQ Regulation (WP7)}

Gaps in aircraft engine emissions regulation will be identified in the causality chain from emissions to ambient and workplace concentrations. WP7 will be the focal point for the AVIATOR project and will aim to identify potential gaps in aircraft emissions regulation. Fig. 5 illustrates the generic inputs and standard setting approaches taken for aircraft engine emissions regulations (left hand side) and local air quality and workplace air quality regulations (right hand side). Therefore, WP7 will build a knowledge framework to help bridge the regulatory approaches.

WP7 will provide coordination, guidance and assistance to WP leaders, to facilitate the translation of technical outcomes into a format and language suitable for the policy and regulatory community, including: (i) ICAO-CAEP and bodies coordinating European input including EASA, and (ii) EEA and EU-OSHA, responsible of providing advice and guidance to European policy makers in setting ambient and workplace air quality standards. Policy orientated technical analyses will seek to further understand and communicate the impact 
with specific reference to PM (nvPM and $\mathrm{vPM}$ ), VOC and SVOC from aircraft engine exhaust emissions on air quality in and around airports.

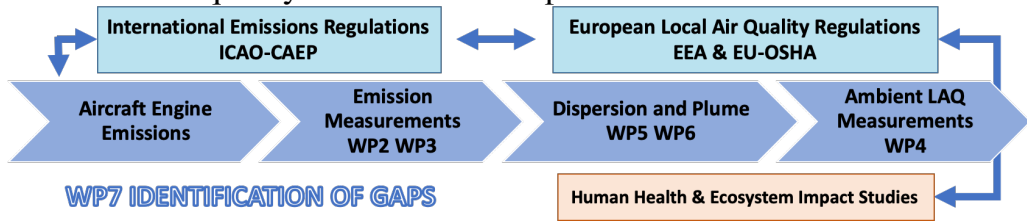

Fig. 5. Potential gaps in aircraft emissions regulation.

AVIATOR will be guided by a multi-sectoral Advisory Board work within the ICAO CAEP process and alongside EASA and industry bodies to ensure that new knowledge, guidance, tools and methods gain maximum traction with the widest set of end users as possible.

AVIATOR Project has received funding from the European Union's Horizon 2020 research and innovation programme under grant agreement No [814801].

\section{References}

1. Air quality in Europe - 2017 report, 13, p. 58, European Environment Agency

2. SAMPLE 3 (EASA), APEX (NASA/EPA), APRIDE4 (FOCA/EASA/FAA) projects.

3. N. Jannsen, C. Ameling, A. Bezemer, O. Breugelmans, A. Dusseldorp, P. Fischer, D. Houthuijs, M. Marra, J. Wesseling, Verkenning gezondheidsrisico's ultrafijnstof luchtvaart rond Schiphol en voorstel vervolgonderzoek, (RIVM Briefrapport, 20160050)

4. ACARE, Strategic Research and Innovation Agenda, 1. See http://www.acare4europe.org/sria

5. ACI EUROPE, Ultrafine Particles at Airports (2018)

6. Ambient Air Quality Directives (2004, 2008). See https://ec.europa.eu/

7. J. Bisson, P. Seers, M. Huegel, F. Garnier, J. Propul. Power, 32, 4, (2016)

8. J.C. Kaiser, J. Hendricks, M. Righi, N. Riemer, R.A. Zaveri, S. Metzger, V. Aquila, Geosci. Model Dev., 7, 3, (2014)

9. J.C. Khou, W. Ghedhaïfi, X. Vancassel, E. Montreuil, F. Garnier, 26, 6, (2017)

10. Lagrangian particle model according to the standard VDI 3945 Part 3. See e.g. U. Janicke, L. Janicke, Lagrangian particle modeling for regulatory purposes; A survey of recent developments in Germany, $11^{\text {th }}$ International Conference on Harmonization within Atmospheric Dispersion Modeling for Regulatory Purposes, (Cambridge, England, 2007). See http://www.harmo.org

11. E. Montreuil, W. Ghedhaïfi, V. Chmielewski, F. Vuillot, F. Gand, A. Loseille, Numerical Simulation of contrail formation on the Common Research Model wing/body/engine configuration, Atmospheric and Space Environments Conference, AIAA 2018-3189, (Atlanta, Georgia, 2018), https://doi.org/10.2514/6.2018-3189

12. Research Project UBA FKZ 3716522000: Influence of a major airport on the temporal and temporal spatial distributions of outside air concentrations of ultrafine particles, German Federal Environmental Agency, (2016-2018)

13. U. Janicke, L. Janicke, LASPORT - A model system for airport-related source systems based on a Lagrangian particle model, $11^{\text {th }}$ International Conference on Harmonization within Atmospheric Dispersion Modeling for Regulatory Purposes, (Cambridge, England, 2007). See http://www.harmo.org 